\title{
Gas detection in sodium cooled fast reactors: determination of a transfer function
}

\author{
C. Ding ${ }^{1}$, P. Filliatre ${ }^{1}$ and L. Desgranges ${ }^{2}$ \\ ${ }^{1}$ CEA, DES, IRESNE, DER, SPESI, LDCI, Cadarache, F-13108 St-Paul-Lez-Durance, France \\ ${ }^{2}$ CEA, DES, IRESNE, DEC, Cadarache, F-13108 St-Paul-Lez-Durance, France \\ congjin.ding@cea.fr
}

\begin{abstract}
To keep the dependability of Sodium Cooled Fast Reactor, the "clean sodium concept" is demanded, which means that the sodium is free from contamination. The release of fission products is searched for by a contamination measuring system. We need to have a comprehensive description of cladding failures and the detection of contamination, including the failure occurrence on the fuel pin, the transfer process through the sodium and cover gas, the measurement efficiency, etc. We aim to identify the important parameters of physical phenomena, with modelling and simulations based on the return of experiments from past reactors such as PHENIX. There have been a total of 15 open pin failures in PHENIX reactor. Through studying these detected signals, we can get a better physical explanation and description of the evolution of failures. The detection system is related to different stages of the evolution of fuel pin, with different types of fission products, various release mechanism and physical properties. During the evolution of the failed fuel pin, gaseous fission products is released on the first stage of failure and the gas detection system is aimed for the gaseous fission products monitoring. We proposed a quantitative modeling of transfer function to describe the time broadening of the gas release from the fuel pin to the detector. The result matches well with PHENIX experiment data, with the same order of magnitude of the time broadening and the same shape of exponential decreasing. A determination of the transfer function of this gas detection system is validated in this paper.
\end{abstract}

Keywords- Sodium fast reactors, PHENIX reactor, cladding failure detection, transfer function

\section{INTRODUCTION}

Fast reactors have the unique capability of improving the usage of natural resources and reducing of volume and heat load of high-level waste thanks to the closed fuel cycle [1]. Among the six types of fast reactor systems, the Sodium cooled Fast Reactor (SFR) has many experience from operating experimental, prototype and commercial size reactors [2].

The "clean sodium concept" is demanded in SFR to keep the dependability of the reactor. A contamination measuring system was designed in order to continuously search for the release of fission products by a fuel pin failure [3]. A fuel pin failure [4] represents a crack or a rupture on the cladding which is the first confinement barrier of the fidel inside the reactor core [5]. Thanks to the 50 years experience of designing, building and operating SFR in France, the fuel pin failure was studied in many fast experimental reactors. For example, during the operational period of the PHENIX reactor (1973-2009), there were a total of 42 gas pin failures, 15 openpin failures [6]. In the detection system of the reactor, gas detection system (DRG system) system is dedicated to detect the failures with gaseous fission products. In this paper, we aim at a more physical interpretation of these data that could be helpful for the design of future designs of SFR.

The transfer process of the detection system is described as a transfer function which convolutes the entry signal to form the observed signal. The main effect of the transfer function is to introduce a time broadening between the fission products released at the pin failure and the detected signal in detectors, which needs to be assessed.

We observe some gas signal in the experimant cases is expected to be nearly instantaneous release according to the gas signal time evolution. It is foreseen that these observed signals are mostly given by the transfer function. We can justfy the assumption of a Dirac peak for this kind of burst and then the the experiment data can be regarded as an experimental evaluation of the transfer function.

The goal of the paper is to set up, using the specification of the PHENIX reactor, a model for the transfer function in order to predict the time broadening of the observed signal. Then we compare the experiment detection to assess how quantitatively predictive the model is. Although the paper will make use of PHENIX data, the model is a general formulation which can be applied to other reactors and to guide the conception choice for the future design.

The paper is organized as follows: the fuel pin failure evolution and the detection system are introduced in Sec. II; the determination of the transfer function is described in Sec. III; the conclusion is summarised in Sec. IV.

\section{FHEL PIN FAILURE AND THE DETECTION-SYSTEM}

In this section, the background of the research is introduced including the evolution of the fuel pin failure and the detection system for gaseous fission products in PHENIX reactor.

\section{A. Evolution of the fuel pin failure}

There are four stages of the fuel pin failure evolution [7], as shown below:

- Normal: This stage is defect-free. The sodium coolant is clean.

- Crack: This stage is only with gaseous fission products. The detection system can only detect gas emissions. During the burn up, most of the gaseous fission products, 
about $80 \%$ to $90 \%$ [8], will be released from the fuel pin and be accumulated in fission gas plenum, which is the gas expansion volume inside the fuel pin. When there is a crack on the cladding, the gaseous fission products in the gas plenum will be released outside the cladding through the crack instantaneously because of the pressure difference between the fuel and the coolant.

- Narrow rupture: A narrow rupture may be subsequent to a crack in case it undergoes several thermal expansions due to various power variations. The detection system can detect neutron signals emitted by delayed neutron precursors. The oxide-sodium reaction (OSR) happens [9].

- Wide rupture: The oxygen atoms feeding the oxidesodium reaction mainly come from the sodium coolant, which continuously flows along the fuel oxide at the rupture location. There are particles of fissile material released into primary circuit. An emergency shut down is immediately ordered.

In summary, gas signal can be detected on the crack stage. If the failure turns to more severe situation, the neutron signal can be detected.

\section{B. Detection system in PHENIX}

The detection system in PHENIX reactor is related to the fuel pin failure evolution, which consists of mainly two parts:

- DRG system (Détection des Ruptures de Gaine) (Gas detection system): DRG system is located in the cover gas. Because gas signal is the first signal can be detected when there is a cladding failure, the aim of DRG system is to know the failure as soon as possible.

- DND system (Détection des Neutrons Différés) (Neutron detection system): DND system is located in the sodium coolant. Because DND signal is concerning the breached fuel area exposed to the coolant, the DND signal is monitored under a threshold.

In addition, there is a LRG (Localisation des Ruptures de Gaine) system for locating the failed pin for both DRG and DND system. This paper will focus on DRG system study. The scheme of DRG-LRG system of PHENIX reactor is shown in Fig. 1. The main detectors for gaseous fission products are shown below:

- MX 32 high activity ionization chambers: they are assigned to the DRG cover gas to supply continuous overall value of the activity in the accident situation.

- Gamma chromatography: it is identification detection and the measurement is periodical (1 time a day) and on demand.

Because MX 32 is the continuous measurement, only the gas signal detected in MX 32 can be applied to transfer function modeling.

\section{DETERMINATION OF THE TRANSFER FUNCTION}

The transfer process of the detection system is described as a transfer function which convolutes the entry signal to form the observed signal. The main effect of the transfer

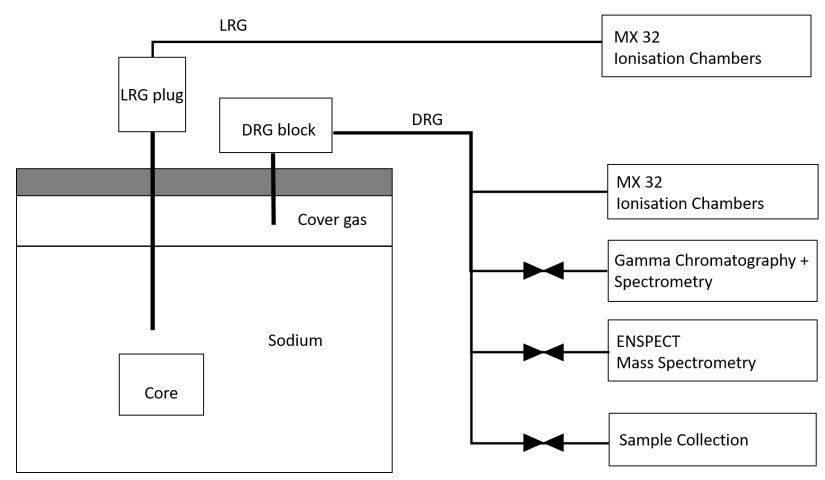

Fig. 1. General scheme of DRG-LRG system in PHENIX reactor.

function is to introduce a time broadening between the fission products released at the pin failure and the detected signal in detectors, which needs to be assessed. To describe the transfer process from the cladding failure to the detector, a scheme of fission gas release is built and a transfer function model is proposed in this section. An experiment case RG 14 in PHENIX reactor with instantaneous release is chosen as an experimental evaluation of the transfer function.

\section{A. Scheme of fission gas transfer and the model of transfer function}

To describe the fission gas release (FGR) transport process from the fuel pin to the detection system, a simplified scheme is built by taking into account fission gas dilution and time broadening.

When there is a crack on the cladding, the fission gas will be released into the sodium coolant circuit rapidly because of the pressure difference. There will be two forms of gas, either in solution in the sodium or in bubbles which eventually go into the cover gas. The solubility of Krypton and Xenon in liquid sodium at $700 \mathrm{~K}$ and $0.1 \mathrm{MPa}$ is calculated by Eq. 1 . According to the Henry's law, the amount of dissolved gas in a liquid is proportional to its partial pressure above the liquid. The amount of dissolved Xenon and Krypton in sodium depends on the partial pressure of Xenon and Krypton above sodium.

$$
x(g) P=x(l) K
$$

In Eq. 1, where $x(g)$ is the number of moles of Xenon and Krypton per number of moles of Argon in the cover gas, $P$ is the absolute pressure above the sodium, $x(l)$ is the number of moles of Xenon and Krypton per number of moles of Sodium, $K$ is Henry constant for Xenon and Krypton, which is derived from the data in [10], which is $4.8 \times 10^{11}$ and $1.4 \times 10^{10}$ respectively.

The calculated fraction of Xenon and Krypton dissolved in sodium is $1.0 \times 10^{-8}$ and $4.0 \times 10^{-7}$ respectively, which are both very low. We can conclude that most of the fission gas will be released into the cover gas. To simplify the transport process, for the predictive calculation we can make 
the hypothesis that all of the FGR goes into the cover gas. Finally the fission gas in cover gas will be sampled into the DRG gas block and be transported to the detection chamber. The activity will be measured by the detectors.

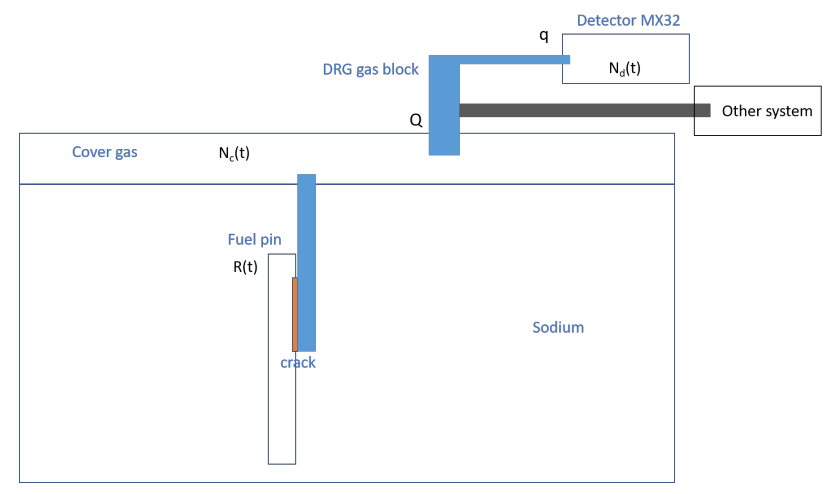

Fig. 2. The scheme of fission gas release from the fuel pin to DRG detection system.

The scheme of FGR from the fuel pin to DRG detection system is shown in Fig. 2. $R(t)$ is the FGR from the fuel pin, in the unit of number of atoms per second. The FGR will go through the crack, which is present in orange in Fig. 2, to the sodium coolant, then will be transported into the cover gas. With the simplification mentioned above, this process is represented by the blue tube leading to the cover gas directly. Then the FGR will dilute in the cover gas. $N_{c}$ is the number of atoms released in cover gas. The total flow rate in the cover gas is $Q$. The sipping system of DRG block samples the FGR from the cover gas, thus the FGR will then flow into the detection chamber, represented by the blue tube leading to the detector MX 32, at the flow rate $q$.

Based on the scheme of FGR from the fuel pin to DRG detection system, we derive the conservation equation of the number of atom of FGR in the cover gas at moment $t$ using the framework of MERARG facility [11], which can be expressed in:

$$
\frac{\mathrm{d} N_{c}}{\mathrm{~d} t}(t)=R(t)-\frac{\square}{V_{c}} N_{c}(t)
$$

The same balance in the detector can be expressed in:

$$
\frac{\mathrm{d} N_{d}}{\mathrm{~d} t}(t)=\frac{q}{V_{c}} N_{c}(t)-\frac{q}{V_{d}} N_{d}(t)
$$

In Eq. 2 and Eq. 3, $R$ represents the FGR from the fuel pin; $Q$ is the total gas flow rate in the cover gas; $q$ is the gas flow rate to the detector $N_{c}$ is the number of atoms released in the cover gas; $N_{d}$ is the number of atom in the detector; $V_{c}$ is the volume of cover gas; $V_{d}$ is the volume of detector chamber.

We can define $q=\alpha Q(0<\alpha<1)$ and apply the Laplace transform to Eq. 2 and Eq. 3, we can express $R$ as a function of $N_{d}$ :

$$
R(s)=\left(\frac{V_{c}}{\alpha Q} s^{2}+\left[\frac{V_{c}}{V_{d}}+\frac{1}{\alpha}\right] s+\frac{Q}{V_{d}}\right) N_{d}(s)
$$

Applying the inverse Laplace transform:

$$
R(t)=\frac{V_{c}}{\alpha Q} \frac{\mathrm{d}^{2} N_{d}}{\mathrm{~d} t^{2}}(t)+\left(\frac{V_{c}}{V_{d}}+\frac{1}{\alpha}\right) \frac{\mathrm{d} N_{d}}{\mathrm{~d} t}(t)+\frac{Q}{V_{d}} N_{d}(t)
$$

We define the transfer function $F(s)$ to express the relation between $R$ and $N_{d}$ :

$$
\begin{gathered}
N_{d}(s)=F(s) R(s) \\
F(s)=\frac{1}{A s^{2}+B s+C}=\frac{1}{A\left[(s+a)^{2}-b^{2}\right]}
\end{gathered}
$$

With $\mathrm{A}=\frac{V_{c}}{\alpha Q}, \mathrm{~B}=\frac{V_{c}}{V_{d}}+\frac{1}{\alpha}, \mathrm{C}=\frac{Q}{V_{d}}, \mathrm{a}=\frac{B}{2 A}, \mathrm{~b}=$ $\sqrt{\left(\frac{B}{2 A}\right)^{2}-\frac{C}{A}}$.

Then we can induce the transfer funtion formula:

$$
f(t)=\frac{1}{A b} \times e^{-a t} \times \sinh (b t)
$$

We can also simplify Eq. 8 and obtain:

$$
f(t)=\frac{\alpha V_{d}}{\alpha V_{c}-V_{d}} \times\left(e^{-\frac{Q}{V_{c}} t}-e^{-\frac{\alpha Q}{V_{d}} t}\right)
$$

The transfer function Eq. 9 is a general formulation of the problem, which is derived from the physical descriptions, can be applied for different detection setups.

For the cases with instantaneous release, we can justify The FGR from the fuel pin is a Dirac peak, then we can obtain:

$$
N_{d}(t)=f(t)
$$

Then we can apply the transfer function to a specific case to compare with the experiment detection to assess how quantitatively predicted the model is.

\section{B. The experiment case $R G 14$}

The RG 14 fuel pin failure occurred on the experimental assembly DCI 3124, SPHINX 5 in 1988. The first gas emission was detected on February 26 at 13 h00. The first major release of fission gas corresponds to the instantaneous leakage of a cladding. Then the internal pressure suddenly drops and the cover gas activity rapidly rises to a maximum of $1.5 \mathrm{Ci} / \mathrm{m}^{3}$ due to the arrival of fission gases.

The signal of the first period fission gas release detected by MX 32 is shown in Fig. 3. We derived the data according to the original curve and the statement in experiment report. We can observe that the gapsignal is expected to be an instantaneous release with time broadening during the first release and then has some time evolution. In order to assess the transfer function, only the first period gas release is studied in this paper.

The post irradiation examination photo for the crack in RG 14 is shown in Fig. 4, taken from the PHENIX internal 


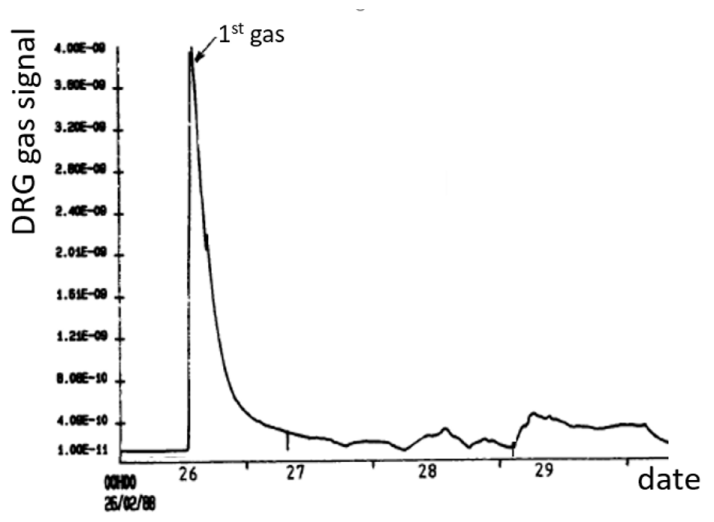

Fig. 3. The signal of the first period fission gas release detected by MX 32 .

report, which is a long and straight breach, with length around $480 \mathrm{~mm}$ and with relatively large width around $0.5 \mathrm{~mm}$. The pin failure happened in the end of life with high burn up of $11.56 \%$, probably due to embrittlement of cladding by the radiation damage. The crack was brittle and large which means the crack occurred rapidly.

For fast reactor, because of the high temperature, steep temperature gradient and the high burn up, the fraction of fission gas release (FGR) in the fission gas plenum is about 80 $\%$ to $90 \%$ [8]. In other words, during the burn up without any failure, most of the gaseous fission products will be released from the fuel pin and be accumulated in fission gas plenum, which is the gas expansion volume inside the cladding. When there is a failure on the cladding, the gaseous fission products in the gas plenum will be released outside the cladding through the crack instantaneously because of the pressure difference between the fuel and the coolant. Thus the crack observation is consistent with the instantaneous gas signal detection.

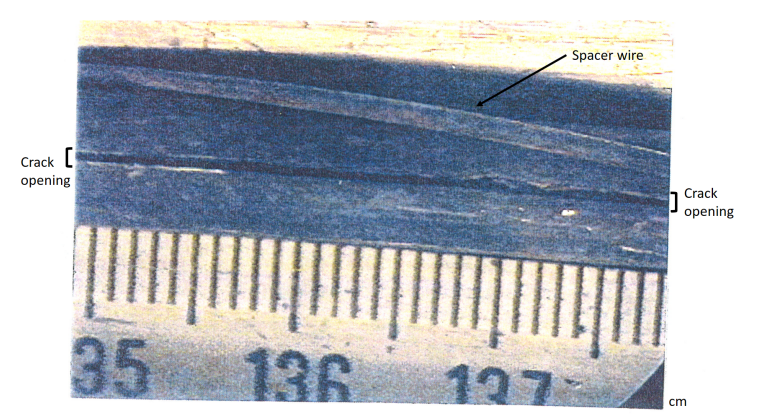

Fig. 4. Post irradiation examination photo (from PHENIX internal report, 24/11/1995).

We can thus justify the assumption of a Dirac peak for the burst release in the case of RG 14. As a consequence, the observed signal of RG 14 can be regarded as an experimental evaluation of the transfer function. Then we can apply the transfer function derived in Sec. III-A to RG 14 case and compare the modeling results with RG 14 experiment data.

\section{Experiment evaluation of transfer function}

In order to do the assessment of the transfer function, the transfer function modeling results and the comparison with the experiment data in RG 14 are shown in this section.

We applied the transfer function to our specific case $R G$ 14 , with the total flow rate in the cover gas $Q(5.0 \mathrm{l} / \mathrm{s})$, the flow rate to MX 32 detector $q(0.2 \mathrm{l} / \mathrm{s})$, as well as volume data $V_{c}\left(40 \mathrm{~m}^{3}\right)$ of the cover gas and $V_{d}(0.09 \mathrm{l})$ in the detector chamber.

As shown in Fig. 5, the red and pink curves are the transfer function modelings taking from Eq. 9, with $Q$ equal to $5.0 \mathrm{l} / \mathrm{s}$ and $1.94 \mathrm{l} / \mathrm{s}$ respectively. Because in accident condition, the flow rate in the cover gas is risen from 1.94 $\mathrm{l} / \mathrm{s}$ to $5.0 \mathrm{l} / \mathrm{s}$.

The blue curve is the experiment data for the gas release detected by MX 32. The X-axis represents the time in hours. To make the comparison, we multiplied a coefficient to make these curves have the same maximum activity value, which is $1.5 \mathrm{Ci} / \mathrm{m}^{3}$. Then we can observe that within a certain flow rate range in the cover gas, the shape of the transfer modeling curve is consistent with the experiment data, which has the same order of magnitude of the time broadening and the same shape of exponential decreasing. We can conclude that the model proposed is able to model the time broadening quantitatively and the transfer function is validated.

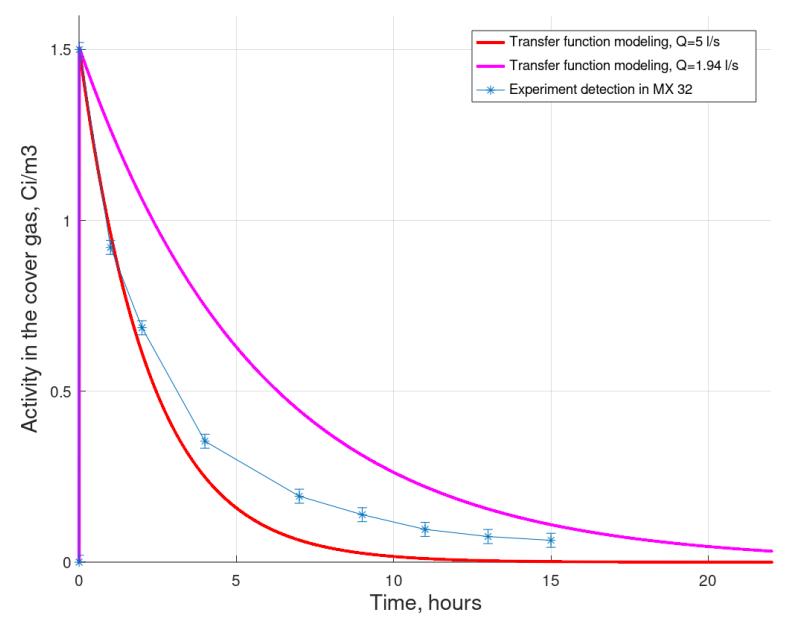

Fig. 5. The fission gas activity in MX 32 and the transfer function modeling results comparison.

\section{CONCLUSIONS}

In this paper, a model for the transfer function of the detection process, making it possible to predict the time broadening of the observed signal, has been proposed. Taking the actual specifications of the reactor, it matches well with the PHENIX reactor experimental data, with the same order of magnitude of the time broadening and the same shape of exponential decreasing. As a result, the transfer function is validated by a comparison between the modeling result and 
the experimental data. Although the study was based on an experimental case in PHENIX reactor, the application for this model can contribute to an improved understanding of fission gas transfer in sodium cooled fast reactor and a guidance for the future design.

\section{REFERENCES}

[1] S.M. Goldberg, R. Rosner, Nuclear Reactors: Generation to Generation, Nuclear Reactors. (n.d.) 40.

[2] G.L. Fiorini, A. Vasile, European Commission - 7th Framework Programme: The Collaborative Project on European Sodium Fast Reactor (CP ESFR), Nuclear Engineering and Design. 241 (2011) 3461-3469. https://doi.org/10.1016/j.nucengdes.2011.01.052.

[3] E. Rohée, R. Coulon, C. Jammes, P. Filliatre, S. Normand, F. Carrel, F. Lainé, H. Hamrita, Delayed Neutron Detection with graphite moderator for clad failure detection in Sodium-Cooled Fast Reactors, Annals of Nuclear Energy. 92 (2016) 440-446. https://doi.org/10.1016/j.anucene.2016.02.003.

[4] J.R. Matthews, T. Preusser, On the failure of fast reactor fuel pins, Nuclear Engineering and Design. 101 (1987) 281-303. https://doi.org/10.1016/0029-5493(87)90056-2.

[5] C. Jammes, N. Chapoutier, P. Filliatre, J.-P. Jeannot, F. Jadot, D. Verrier, A.-C. Scholer, B. Bernardin, Neutron flux monitoring system of the French GEN-IV SFR: Assessment of diverse solutions for invessel detector installation, Nuclear Engineering and Design. 270 (2014) 273-282. https://doi.org/10.1016/j.nucengdes.2013.12.057.

[6] D. Tenchine, Some thermal hydraulic challenges in sodium cooled fast reactors, Nuclear Engineering and Design. 240 (2010) 1195-1217. https://doi.org/10.1016/j.nucengdes.2010.01.006.

[7] Herbreteau, K., Marie, N., Bertrand, F., Seiler, J.-M., Rubiolo, P., 2018. Sodium-cooled fast reactor pin model for predicting pin failure during a power excursion. Nuclear Engineering and Design 335, 279-290. https://doi.org/10.1016/j.nucengdes.2018.05.023

[8] C.A. Friskney, J.A. Turnbull, The characteristics of fission gas release from uranium dioxide during irradiation, Journal of Nuclear Materials. 79 (1979) 184-198. https://doi.org/10.1016/0022-3115(79)90446-X.

[9] Barkia, B., Courouau, J.L., Perrin, E., Lorentz, V., Rivollier, M., Robin, R., Nicolas, L., Cabet, C., Auger, T., 2018. Investigation of crack propagation resistance of $304 \mathrm{~L}, 316 \mathrm{~L}$ and $316 \mathrm{~L}(\mathrm{~N})$ austenitic steels in liquid sodium. Journal of Nuclear Materials 507, 15-23. https://doi.org/10.1016/j.jnucmat.2018.04.036

[10] E.E. Shpil'rain, S.N. Skovorod'ko, A.G. Mozgovoi, New Data on the Solubility of Inert Gases in Liquid Alkali Metals at High Temperature, 40 (n.d.) 7.

[11] P. Menegon, L. Desgranges, Y. Pontillon, A. Poulesquen, Evidence of two gas release kinetics during the oxidation of an irradiated PWR UO2 fuel, Journal of Nuclear Materials. 378 (2008) 1-8. https://doi.org/10.1016/j.jnucmat.2008.04.008. 